\section{Apertura al conocimiento en Ciencias Naturales a través de la experimentación}

Mirta Furlani

mfurlani@fiq.unl.edu.ar

Susana Palma

spalma@fiq.unl.edu.ar

Silvina Rebechi

srebechi@fiq.unl.edu.ar
María Sara Salsi

msalsi@fiqus.unl.edu.ar

Docentes investigadoras de la

Facultad de Ingeniería Química.

Universidad Nacional del Litoral,
Argentina.
Integración de la docencia y la extensión /

Intervenciones

RECEPCIÓN: 24/06/16

ACEPTACIÓN FINAL: 10/10/16

\section{Resumen}

El acercamiento de un grupo de docentes universitarios a la enseñanza y al aprendizaje de las Ciencias Naturales del nivel medio mediante cursos de formación docente, fue el disparador por el que la Universidad Nacional del Litoral y una escuela media de la ciudad de Santa Fe se vincularan a través de un Proyecto de Extensión de Cátedra. El proyecto consistió en generar estrategias sobre base de la experimentación como una forma de "aprender y enseñar ciencias" a partir de la puesta a punto del equipamiento disponible en las escuelas que no había sido usado con anterioridad y de transmitir esta experiencia a la comunidad.

\section{Resumo}

A aproximação de um grupo de docentes universitários ao ensino e aprendizagem das Ciências Naturais do ensino médio através de cursos de formação docente, foi o estopim para a Universidad Nacional del Litoral e uma escola da cidade de Santa Fe se vincularem a partir de um projeto de extensão de disciplina. O projeto consistiu em gerar estratégias sobre a base de experimentação como uma forma de "aprender e ensinar ciências" a partir da vistoria do equipamento disponível nas escolas que não tinha sido usado com antecedência, e de transmitir esta experiências à comunidade.

Palavras-chave

- Ciências naturais

- Educação experiencial

- Estratégias didáticas

- Experiências de laboratório

- Escola de ensino médio
Para citación de este artículo

Furlani, M.; Palma, S.; Rebechi, S. y Salsi, M.S. (2016). Apertura al conocimiento en ciencias naturales a través de la experimentación. En Revista +E versión digital, (6), pp. 352-359. Santa Fe, Argentina: Ediciones UNL. 


\section{Introducción}

El proyecto consistió en abordar la problemática de la puesta en funcionamiento de un laboratorio de Ciencias Naturales Básicas en la Escuela de Educación Técnica n 647 Pedro Lucas Funes (EET), que se encuentra emplazada en el cuadrante noreste del barrio Centenario de la ciudad de Santa Fe.

Los antecedentes de este proyecto se originan en el año 2007 a raíz del vínculo de algunos docentes de la Facultad de Ingeniería Química de la Universidad Nacional del Litoral (FIQ-UNL) con una ONG local (la Asociación para la Investigación y la Docencia Universitas Argentina) se generó una propuesta de curso de capacitación para docentes del nivel medio aprobado por la Red Federal de Formación Continua del Ministerio de Educación de la Provincia de Santa Fe (RM n ${ }^{\circ}$ 617/07). La sede de dicho curso fue la FIQ. Una treintena de docentes secundarios de distintos establecimientos del nivel medio de la ciudad de Santa Fe y localidades vecinas se involucraron en ello y evaluación final consistió en el desarrollo y exposición de un trabajo experimental de laboratorio, que fue defendido ante los docentes del curso y sus propios compañeros. Dos docentes de ese curso, uno de ellos estudiante de Ingeniería Química, fueron el vínculo para que luego se gestaran dos proyectos de extensión de cátedra.

La propuesta de trabajo que se presentó entonces surgió ante una necesidad objetiva y real en un espacio y tiempo definidos. Es decir, se dio como un acontecimiento en torno al quehacer educativo de un grupo de personas y no solo como la vinculación de estructuras que se nuclean para un determinado fin. Docentes y directivos de la mencionada institución educativa vislumbraron que la articulación con la actividad académica universitaria sería relevante al momento de intentar algunas acciones de colaboración que aún no estaban definidas, que no se sabía cómo encauzarlas y para las que se intentaba buscar una respuesta. Tuvieron en consideración uno de los objetivos de la educación media, que establece articular la oferta educativa con diferentes tipos de instituciones y que la Universidad, desde los distintos ámbitos de su propia organización, responde con particular interés al facilitar vínculos con la sociedad, con otros niveles educativos y con personas que perciban como necesarias la permanente capacitación y la integración en diversos aspectos culturales, educativos y productivos. Fue entonces que la Secretaría de Extensión de la UNL realizó una convocatoria y el Proyecto de Extensión de Cátedra (PEC) se ajustó a esta demanda de brindar apoyo en el diseño y puesta a punto de experiencias de laboratorio y estrategias didácticas para la experimentación en el área de las Ciencias Naturales.

En el establecimiento educativo existía un espacio físico destinado al laboratorio. No obstante esto, una de las dificultades que manifestaron estos profesores fue que toda la comunidad educativa no estaba de acuerdo en cómo hacer uso del mismo. Al no darle el uso al que estaba destinado y con la frecuencia requerida, con el correr del tiempo dicho ámbito fue ganado por el desarrollo de otro tipo de actividades que no estaban relacionadas con experiencias en ciencias.

Al momento de conocer el emplazamiento del establecimiento educativo del nivel medio, sus instalaciones y el espacio físico destinado al laboratorio, se observó la existencia de cajas modulares con diversos equipos para experiencias de Física, material de vidrio, centrífugas, reactivos de laboratorio, microscopios, aparentemente en buenas condiciones para desarrollar una importante diversidad de trabajos experimentales. Dicho equipamiento no se había usado hasta esa fecha y había sido afectado por las inclemencias de la gran inundación de 2003, con consecuencias que nunca habían sido evaluadas. Esto implicó un meticuloso relevamiento para constatar qué partes estaban en buenas condiciones y cuáles debían repararse o cambiarse. 
El principio de "extender" la presencia de la Universidad en la sociedad se constituye también, y principalmente, en el contexto de un PEC y de las personas que participan en el mismo en una acción educativa. Giussani (2004) señala que:

"la educación consiste en una introducción a la realidad total" y que "es interesante advertir el doble valor de ese 'total': educación significa el desarrollo de todas las estructuras del individuo hasta su realización integral, y al mismo tiempo, la afirmación de todas las posibilidades de conexión activa de esas estructuras con la realidad".

La educación, entendida como apertura a toda la realidad, implicó en nuestro accionar tener en cuenta los múltiples factores en juego y las potencialidades humanas de docentes, alumnos, familias y entorno social, para gestar un entramado humano que supiera vencer las dificultades en pos de responder a las preguntas: ¿Cuál es el significado explicativo del vínculo entre la Universidad y la sociedad, que aflora en la experiencia? ¿Qué sentido tiene tender a generar este entramado de relaciones con personas que transitan distintos ámbitos cotidianos?

Para dar respuesta a estas preguntas y para mantener el vínculo con la comunidad de la escuela media, se concretaron dos PEC durante dos años. El primero fue: "Identificar la estrategia óptima para la puesta en funcionamiento de un laboratorio de Ciencias Experimentales Básicas"1 y el segundo: "Asombro y realidad. Las Ciencias Naturales en acción". ${ }^{2}$

\section{Perspectivas de los PEC}

Gustavo Menéndez señala que:

"La extensión es un proceso de comunicación entre la universidad y la sociedad basado en el conocimiento científico, tecnológico, cultural y en su capacidad de formación educativa con plena conciencia de su función social” (2004:1).

Este proceso de comunicación tiene implicancias primordiales y específicas que enriquecen la extensión universitaria. Para docentes y alumnos universitarios implicados significó proyectar los saberes aprendidos haciéndolos significativos para alumnos y docentes del nivel medio y su entorno, explorar formas de acercamiento y valorar las realidades culturales encontradas. Se procuró que los alumnos del nivel medio alcanzaran conocimientos y prácticas vinculadas a la experimentación en ciencias básicas; mientras que para el medio social en que residen los alumnos se propuso, a través de la divulgación científica, que accedieran a adelantos tecnológicos. Para los docentes del nivel medio implicó favorecer la capacitación permanente.

Camilloni menciona que "la educación experiencial es una estrategia de enseñanza destinada a relacionar el aprendizaje académico con la vida real” (2013:15), en la que los estudiantes universitarios aplican sus habilidades y conocimientos académicos para satisfacer necesidades concretas en respuesta a requerimientos explícitos de la comunidad. Además de servir a su futura formación profesional, Camilloni indica que:

"[el] aprendizaje-servicio, pone el acento en los dos términos y no sólo en uno de ellos; contribuye a la educación para la ciudadanía y a su formación ética, diferenciándose así, de la práctica profesional que se centra en el aprendizaje mientras que el voluntariado lo hace en el servicio". (Camilioni, 2013: 17-18)

La dimensión pedagógica de la educación experiencial se pone de manifiesto de manera significativa en los procesos de enseñanza y de aprendizaje ya que son instrumentos para crear situaciones y abordar contenidos que permiten al alumno vivir experiencias para su propia transformación personal. Por ejemplo, en una situación en la cual corresponde compartir una actividad con aquellos a quienes va dirigida la extensión, se requiere un real compromiso humano para priorizar qué necesidad tiene la otra persona en ese momento mediante el empleo de un lenguaje accesible y una comunicación abierta. El estudiante universitario durante el servicio reconvierte el conocimiento de los contenidos teóricos y prácticos al confeccionar guías de trabajos experimentales y colaborar con los docentes secundarios en la redacción de la planificación áulica, y es allí donde realiza la transposición didáctica al adaptar y poner en contexto los contenidos. Otro rasgo de la educación experiencial se encuentra en la solución de problemas reales, como se dio en la puesta a punto de trabajos prácticos experimentales con equipamiento no usado. Allí fue primordial la atención ya que se debió generalizar y adaptar lo aprendido para una situación diferente y en este caso multidisciplinaria y multiprofesional. Se trató de un proceso integrado donde es de suma importancia transformar el conocimiento en acción. 
El docente universitario que guía al alumno extensionista asume la tarea de proponer consignas, orientar la modelización del problema sobre el que se trabaja y diseñar la evaluación. Se propuso una evaluación en dos dimensiones:

a) Continua, acompañando la experiencia de cada alumno universitario, ofreciendo espacios de confrontación orientadora de las observaciones e interpretaciones que realice en forma personal, así como de la modalidad con la que resuelve problemas pertinentes a los temas desarrollados y actividades propuestas. Esta evaluación se implementará a través de las siguientes actividades:

- La solución de problemas reales como fue el diseño, armado y puesta a punto de equipos para experimentación, con material no usado.

- El diseño y puesta a punto de trabajos prácticos, confección de guías y la generación de estrategias para la formación docente.

- La observación de clases y seguimiento de los alumnos del nivel medio.

- La redacción del cuaderno de bitácoras por parte de todos los alumnos universitarios.

b) Integradora, a través de un breve informe final de las actividades realizadas. Y la presentación de una guía de trabajo práctico, original, confeccionada en forma grupal entre dos o tres alumnos, cuyo tema fue acordado previamente.

Los alumnos del nivel medio fueron evaluados según las normas vigentes en ese nivel educativo y por sus propios docentes.

\section{Proceso de formulación del problema y objetivos}

Como se mencionó, identificar una necesidad y buscar el camino más adecuado para dar respuesta a la misma hasta definirla como un problema de extensión y justificar su utilización como recurso pedagógico para la planificación de una dada asignatura no son acciones inmediatas. Es más bien un proceso complejo que difícilmente se pueda describir en una serie de pasos o en un esquema preconcebido. En este contexto el equipo que elaboró la propuesta, con experiencia docente en la Universidad, procuró delimitar la situación problema.

En primer lugar, se buscaron las coincidencias entre la formación disciplinar de los docentes universitarios participantes y los alumnos universitarios que cursaban determinadas asignaturas: Física II; Química, Nutrición y Legislación de Alimentos; Química Analítica Aplicada a Alimentos en la FIQ-UNL, las materias que se dictan en el nivel medio entre $1^{\circ}$ y $5^{\circ}$ año y los respectivos alumnos y docentes, dando prioridad a las ramas del conocimiento Química, Física y Biología de las Ciencias Naturales.

En segundo lugar, para avanzar hacia la definición de los objetivos se tomó en cuenta que, si bien la problemática de nuestro interés es común observarla en cualquier colegio del nivel medio, limitamos nuestro accionar al nivel medio técnico y los alumnos beneficiarios de este proyecto concurrían a una institución educativa ubicada en una zona geográficamente periférica y caracterizada en lo social por una población estudiantil con nivel económicamente careciente. Con estas consideraciones hemos llegado a plantear los siguientes objetivos:

- Adaptar el espacio físico disponible en la escuela para la experimentación y la divulgación en Ciencia Naturales, relevando el material disponible y puesta a punto de experiencias sencillas en función del equipamiento encontrado.

- Introducir los espacios de las Ciencias Naturales a partir de experiencias de la vida diaria abordando la Química, Física y la Biología en acción.

- Transferir al medio experiencias sencillas para ser presentadas durante la Exposición Anual de Trabajos.

\section{Etapas}

Ambos proyectos se llevaron a cabo teniendo en cuenta los aspectos de la difusión a los interesados; el relevamiento del material disponible en la EET y la búsqueda bibliográfica; la puesta a punto solamente de experiencias de Física en el primer proyecto y de experiencias de Física, Química y Biología en el segundo proyecto; la confección de guías para realizar los experimentos y el desarrollo de estrategias docentes para el dictado de las clases experimentales. Todo esto se planteó en etapas sucesivas, orientativas, sin que signifique un accionar poco flexible sino más bien el mismo se fue adaptando a las necesidades que la realidad exigía.

\subsection{Etapa de divulgación}

Esta etapa es de primordial importancia para la concreción posterior del proyecto, en una forma armoniosa y articulando también el interés de quienes, sin estar directamente vinculados al proyecto, constituyen un círculo más externo al mismo. Despertar el estupor de los alumnos y docentes por algo que es una novedad requiere de especial atención y cuidar con esmero los pasos que se van dando. Estos pasos fueron la confección de carteles, invitación verbal y mediante correo electrónico a reuniones explicativas dirigidas a los alumnos universitarios que ese cuatrimestre cursaban las asignaturas involucradas en el PEC, como también a alumnos que ya habían cursado el cuatrimestre anterior y que podían interactuar por sus conocimientos consolidados.

En un principio se presentaron el título, los objetivos, los plazos estipulados para su ejecución y las etapas del posible accionar posterior.

\subsection{Etapa de desarrollo}

En general, los alumnos universitarios que libremente decidieron vincularse al proyecto desconocían el emplazamiento, sus 


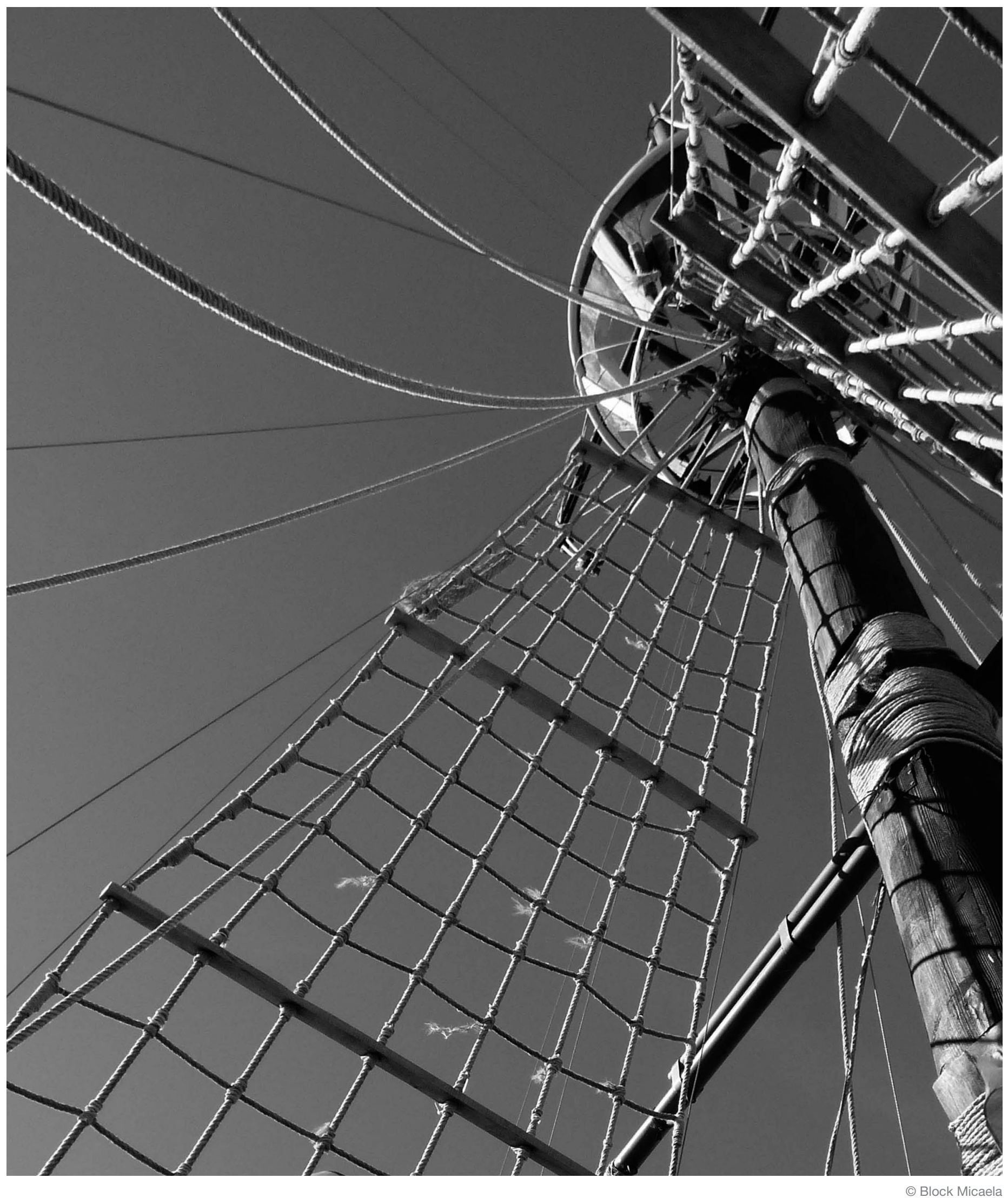


características y hasta cómo llegar a la EET. Todo ello constituyó un aprendizaje en paralelo. Esto condujo a evaluar si existían posibles riesgos para los alumnos en los trayectos de ida y vuelta desde un lugar a otro y viceversa. En consecuencia, debimos precisar los horarios de apertura y cierre de la EET, de modo que siempre hubiese una persona que facilitara el acceso al sector áulico destinado al laboratorio. De igual modo, siempre iban acompañados por docentes de la Facultad para supervisar y guiar las actividades.

Por otra parte, una tarea primordial es recuperar permanentemente los objetivos del proyecto. Para esto fue necesario realizar reuniones con cierta periodicidad para reconocer que a todos no movía el mismo interés, recuperar lo positivo del accionar hasta ese momento y proyectarse hacia posibilidades aún no concretadas.

\subsection{Etapa de análisis de las acciones en proceso}

Integrar las acciones de extensión al proceso de enseñanza y de aprendizaje e involucrar a los alumnos en la realización de trabajos prácticos conllevaron la capacidad de comprender la importancia de la educación experiencial al relacionar el aprendizaje académico con la vida real.

La puesta a punto de trabajos prácticos contempló el planteo del problema y la selección de los métodos adecuados para su solución. Se planteó la adaptación armónica de la bibliografía, del material disponible y de la planificación escolar. Se respetaron las normas básicas de seguridad en el laboratorio.

Se sugirió reiteradamente a los alumnos universitarios la continuidad en la redacción del cuaderno de bitácora.

Se orientó a los alumnos secundarios a poner en juego su potencialidad para abordar objetivos relacionados con el aprendizaje de conocimiento conceptual y procedimental, aspectos relacionados con la metodología científica y la promoción de capacidades de razonamiento (pensamiento crítico y creativo).

\section{Cambios efectivamente producidos}

La participación de los alumnos universitarios consistió en el relevamiento del material de laboratorio, la búsqueda bibliográfica, el armado de equipos, la puesta a punto de trabajos prácticos, la confección de guías para el desarrollo de los mismos, la observación de clases y el seguimiento de los alumnos del nivel medio durante el dictado de las clases. La puesta a punto de trabajos prácticos implicó la adquisición de datos experimentales y análisis de los mismos, la propuesta de un modelo matemático sencillo y la verificación experimental de alguna relación entre magnitudes fisicoquímicas.

Con respecto a los alumnos del nivel medio, se pudo mejorar no solo su calidad científico-pedagógica en la observación, descripción y comprensión de fenómenos de las Ciencias Naturales y la confección de informes sencillos sino también su capacidad para tomar iniciativas e involucrarse en preguntas y problemas.

Respecto de los docentes del nivel medio, se los pudo acompañar en el dictado de los trabajos prácticos a sus alumnos y se les propuso estrategias para la formación docente con una apertura a la experimentación, tales como el "aprendizaje activo", el desarrollo de "pequeñas investigaciones" y una propuesta desde un "enfoque interdisciplinario". En relación con las estrategias de enseñanza sobre la base de la experimentación y como una forma de "aprender a enseñar ciencias", nos remitimos a nuestra experiencia personal en la enseñanza, en la ejercitación de la ciencia y en nuestro continuo aprender a enseñar los procedimientos de las ciencias experimentales.

Siendo que tradicionalmente las llamadas prácticas de laboratorio siempre se han considerado un pilar fundamental de la enseñanza de las ciencias, se logró que tuvieran un espacio físico definitivo para este tipo de prácticas una vez asimilada la importancia de estas actividades. También quedaron en las escuelas guías de distintos trabajos prácticos para los alumnos con aspectos didácticos y fundamentos teóricos para los docentes.

Los alumnos universitarios del Profesorado de Química que participaron activamente de este proyecto también vivieron una experiencia especial y algunos se manifestaron de la siguiente manera:

Alumno 1: - "Creo que mi participación en este tipo de proyectos es muy importante tanto a nivel académico como personal.

Me permitió poner en práctica los conocimientos que vamos adquiriendo, incorporar conocimientos nuevos y conocer distintas realidades. De algún modo nos proyecta como futuros profesionales siendo consciente de la realidad que nos rodea y nos permite crecer como personas".

Alumno 2: - "La participación en el proyecto resultó una experiencia de aprendizaje en todo sentido que va desde descubrir las bases para realizar investigaciones, el trabajar en grupo, desarrollar un criterio de trabajo, el experimentar y lo más importante el aportar algo a la sociedad".

Alumno 3: - "Pude comprobar que la relación entre distintas instituciones, como en este caso la Escuela Técnica y la FIQ, es posible a través de personas concretas, si hay algunos objetivos comunes. Responder a una necesidad objetiva tanto de alumnos como docentes de otra institución educativa puede ocasionar el despertar del interés personal por la propia formación, y el interés de la ayuda a otros en su propio ambiente. Mi formación como estudiante de una carrera universitaria es útil al momento de aplicar lo aprendido en situaciones diversas".

En la siguiente Tabla se presentan las distintas experiencias implementadas en función del equipamiento, material y reactivos disponible en la escuela: 


\begin{tabular}{|c|c|c|}
\hline Material disponible & Actividad curricular experiencia de & Descripción \\
\hline Microscopios, porta y cubre objetos. & Química, Biología y Física & $\begin{array}{l}\text { Observación de gránulos de almidón y polen. Observación de bacterias de yogur. } \\
\text { Marcha de rayos en el microscopio. }\end{array}$ \\
\hline Centrífuga, balanza analítica. & Química & Desnaturalización de las proteínas de la leche y obtención de suero. \\
\hline Equipo de destilación. & Química & Armado y destilación de una muestra de vino para extracción de alcohol. \\
\hline $\begin{array}{l}\text { Morteros, embudos, papel de filtro, } \\
\text { tubos de centrífuga, alcohol etílico. }\end{array}$ & Química & Extracción de pigmentos naturales de las plantas. Cromatografía en papel. \\
\hline $\begin{array}{l}\text { Tubos de ensayos, vasos de } \\
\text { precipitados. }\end{array}$ & Química & $\begin{array}{l}\text { Reacciones para diferenciar pigmentos naturales. Extracción de colorantes } \\
\text { artificiales y cromatografía con tizas. }\end{array}$ \\
\hline Erlenmeyer. & Biología & Crecimiento de levadura biológica para obtención de gas carbónico. \\
\hline Estufa de cultivo y cajas de Petri. & Biología & Observación de crecimiento de colonias. \\
\hline Hidróxido de sodio, sulfato de cobre. & Química & Reconocimiento de la presencia de proteínas por ensayo de Buiret. \\
\hline lodo, ioduro de potasio. & Química & Reconocimiento de almidón en alimentos. \\
\hline $\begin{array}{l}\text { lodato de potasio, almidón, verde } \\
\text { de malaquita. }\end{array}$ & Química & Detección de sulfito en jugos y carnes. \\
\hline lodo y almidón. & Química & Detección de la presencia de vitamina C en jugos. \\
\hline Riel para movimientos de móviles. & Física & Movimiento rectilíneo. \\
\hline Lentes y espejos. & Física & Óptica. \\
\hline Circuitos en serie. & Física & Electricidad. \\
\hline Bobinas e imanes. & Física & Magnetismo. \\
\hline
\end{tabular}

\section{6}

\section{a lo largo de estos proyectos los alumnos universitarios se consolidaron como} equipo al reforzar su compañerismo; y en la interactuación con los docentes secundarios hicieron una experiencia docente importantísima

\section{Conclusiones}

En estas conclusiones vamos a resaltar el positivo impacto de estos proyectos en la comunidad educativa de la Escuela de Educación Técnica n 647 Pedro Lucas Funes, cuyos destinatarios fueron alumnos adolescentes, de $1^{\circ}$ a $5^{\circ}$ año que sumaron un total de 250, docentes del nivel medio vinculados a las Ciencias Naturales, directivos y comunidad en la que está inserta la escuela. Se logró adaptar el espacio físico que ya existía y fue destinado exclusivamente para laboratorio. Asimismo, se planificó su utilización en forma frecuente mediante la implementación de trabajos prácticos con el material existente. También en el marco de estos proyectos se realizaron experiencias interactivas para los alumnos de la escuela y de $7^{\circ}$ grado de escuelas vecinas durante la exposición anual. Lo manifestado hasta aquí ya responde las preguntas planteadas en la introducción.

Por otra parte, a lo largo de estos proyectos los alumnos universitarios se consolidaron como equipo al reforzar su compañerismo; y en la interactuación con los docentes secundarios hicieron una experiencia docente importantísima, en tanto pudieron volcar sus conocimientos pedagógicos en la práctica diaria, actuando como futuros docentes. La positividad de esta experiencia se verificó en la buena recepción de los distintos participantes involucrados, como se pudo leer en las reflexiones 


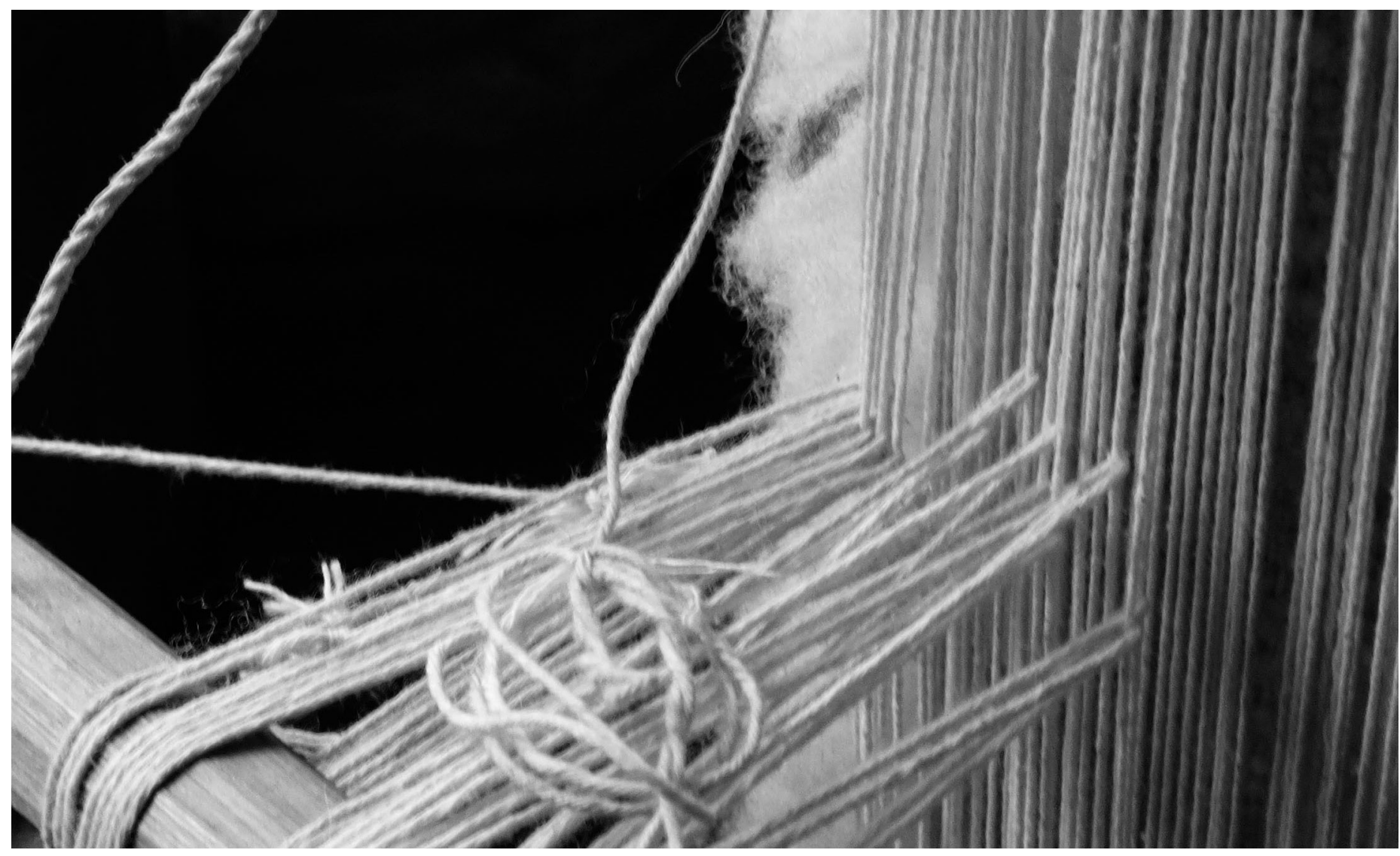

(c) Block Micaela

antes expuestas. Los alumnos del Profesorado de Química que participaron del PEC fueron coautores con sus docentes de una comunicación que refiere a la exposición anual de trabajos de la escuela técnica en las $X$ Jornadas Nacionales y VII Jornadas Internacionales de Enseñanza de la Química Universitaria, Superior, Secundaria y Técnica de la Asociación Química Argentina en Buenos Aires en 2015.

Con respecto a la experimentación, es ampliamente admitido, tanto por investigadores como por educadores, que el trabajo de laboratorio debe ser un componente fundamental de la enseñanza y del aprendizaje de las ciencias, especialmente durante la escolaridad básica. Dicha actividad consiste en el uso de material de laboratorio para reproducir un fenómeno o para analizar una parte del mundo a estudiar. La ausencia de dicha actividad conduce a una educación academicista, retórica y desvinculada del fenómeno físico real y por lo tanto descontextualizada de la totalidad de los factores implicados en los procesos de enseñanza y de aprendizaje de los fenómenos de las ciencias experimentales. En relación con el equipamiento de laboratorio, se propone que responda al requisito de robustez en su fabricación para contribuir a la confiabilidad y contundencia de los resultados a la vista de los alumnos del secundario.
En cuanto a las estrategias para la formación docente en una apertura a la experimentación, se puede concluir que, refiriéndose al enfoque interdisciplinario, los estudiantes del nivel medio manifestaron preferir esta combinación ya que en el contexto de la Química y la Biología se hacen más significativas las cuestiones físicas.

Además, es conocido que hay escasez de proyectos institucionales que tiendan a poner en funcionamiento los laboratorios para las Ciencias Naturales en el nivel medio.

Es esperable que se generalice la realización de experiencias de laboratorio de Física, de Química y de Biología en nuestro país y para ello se requiere una mirada nueva sobre la enseñanza y el aprendizaje de las Ciencias Naturales.

\section{Referencias bibliográficas}

Giussani, L. (2004). El Riesgo Educativo. Buenos Aires: Ciudad Nueva.

Menéndez, G. (2004). Desarrollo y conceptualización de la extensión universitaria. Un aporte de la Secretaría de Extensión de la Universidad Nacional del Litoral para al análisis y debate acerca de la Extensión Universitaria. Santa Fe: Ediciones UNL. Menéndez, G. y otros (2013). Integración docencia y extensión. Otra forma de aprender y de enseñar. Santa Fe: Ediciones UNL. 